\title{
La psicología y su influencia en el clima laboral de las empresas
}

\section{Psychology and its influence on the work environment of companies}

Washington Edy Santillán Marroquín. 1, Hernán Eduardo Paredes García. ${ }^{2}$, Galo Edmundo Garcés Uquillas. ${ }^{3}$ Josué Fernando Santillán Vieira. $^{4}$

\section{Abstract. $\quad$ DOI: https://doi.org/10.33262/cienciadigital.v3i3.2.757}

To integrate Psychology (organizacional and clinical), to propose other practices to improve the LABOR CLIMATE of the Organizations; that it includes, Organizacional Psychology with its specific processes and the wealth of Clinical Psychology using the model of the Familiar Therapy Sistémica for the families of the workers of a Company, is the proposal of this article. With the Familiar Therapy we allowed ourselves to suggest other alternatives of management that leads to a process of continuous improvement of the labor climate of the Organizations, using information, tools and strategic innovators. We will treat to demonstrate that these fields of the psychology can be used harmonically, on the one hand, so that the professionals of clinical psychology have a powerful tool in the familiar therapy and that she serves to improve the labor climate of the organizations, and on the other hand, so that the organizacionales psychologists, find new strategies and other innovating practices, in the improvement of this labor climate, that not necessarily is the qualification, education, development of leaders and training of the personnel, but, resort to other processes such as; the emotional wage, political familiarly responsible, RSC Corporative Social Responsibility or other similars, stop "to obtain" important changes in the families and the collaborators who conform and who as well, are fundamental part of a good atmosphere in the companies in the era of the talentismo.

\footnotetext{
${ }^{1}$ Instituto Ohiggins, Quito Ecuador, coordinacion.academica@instituto-ohiggins.com 2Instituto Ohiggins, Quito Ecuador, coordinacion.academica@instituto-ohiggins.com ${ }^{3}$ Instituto Ohiggins, Quito Ecuador, garcesgalo@hotmail.com ${ }^{4}$ Universidad Internacional SEK Ecuador,Quito, Ecuador. jsantillan.mgth@uisek.edu.ec
} 
Keywords: Work climate, continuous improvement, systemic family therapy, innovative strategies, new practices, families, collaborators.

\section{Resumen}

Integrar la Psicología (organizacional y clínica), proponer otras prácticas para mejorar el CLIMA LABORAL de las Organizaciones; que incluye, la Psicología Organizacional con sus procesos específicos y la riqueza de la Psicología Clínica utilizando el modelo de la Terapia Familiar Sistémica para las familias de los trabajadores de una Empresa, es la propuesta de este artículo. Con la Terapia Familiar nos permitimos sugerir otras alternativas de gestión que nos conduzca a un proceso de mejora continua del clima laboral de las Organizaciones, utilizando información, herramientas y estratégicas innovadoras. Trataremos demostrar que estos campos de la psicología se los puede utilizar armónicamente, por una parte, para que los profesionales de la psicología clínica tengan una herramienta poderosa en la terapia familiar y que sirva para mejorar el clima laboral de las organizaciones, y por otro lado, para que los psicólogos organizacionales, encuentren nuevas estrategias y otras prácticas innovadoras, en la mejora de dicho clima laboral, que no necesariamente sea la capacitación, educación, desarrollo de líderes y entrenamiento del personal, sino, recurran a otros procesos tales como; el salario emocional, políticas familiarmente responsables, RSC Responsabilidad Social Corporativa u otros similares, para "lograr" cambios importantes en las familias y en los colaboradores que conforman y que a su vez, son parte fundamental de un buen ambiente en las compañías en la era del talentismo.

Palabras Claves: Clima laboral, mejora continua, terapia familiar sistémica, estrategias innovadoras, nuevas prácticas, familias, colaboradores.

\section{Introducción.}

Este tema tiene que ver con una nueva propuesta, un modelo en el que se evidencia, el impacto de la terapia familiar y otros procesos modernos de la gestión del Talento Humano y que se encuentran vigentes, para mejorar de manera sostenida el clima laboral en las organizaciones, se puede definir como la integración "ideal" de las dos ramas de la Psicología, (Organizacional y Clínica), y que están relacionadas de forma sistémica, ya que estudian e integran dos sistemas o subsistemas pocorelacionados en la práctica diaria y que deberían conciliarse, como son las familias y las empresas (Centro Walmart, 2014).

Los psicólogos industriales u organizacionales, no sólo se ocupan de sus procesos tradicionales tales como: evaluación al desempeño, selección e inducción, entrenamiento, capacitación, plan de carrera, análisis de clima laboral, evaluación de cargos, incentivos, condiciones de trabajo, nómina, beneficios, servicios, competencias e indicadores de gestión etc., que por lo general tienen una gran carga administrativa, sinotambién, del 
comportamiento de los grupos y subsistemas de trabajo y de la respuesta de la organización en su conjunto ante estímulos internos y externos, generando las "Buenas Prácticas Laborales" con un enfoque más psicológico, intelectual y emocional (Juape, 2014).

Esta actividad pretende fomentar el trabajo interdisciplinario para ir acorde con los paradigmas actuales con un tinte holístico e integral.

Por otro lado, existen diferentes orientaciones teóricas entre los profesionales que intervienen en terapia familiar. Nuestra primera elección en esta propuesta, es de un enfoque familiar sistémico y estructural que corresponde a nuestra convicción de que dicho modelo es eficaz para el tratamiento institucional y clínico. Sin embargo, incluyo otros criterios de orientación organizacional y que vale la pena puntualizarlos en esta investigación (Minuchín, 1986; Fineman, 2000).

Por lo tanto, las Organizaciones para mejorar su Clima laboral requieren "Romper Paradigmas" y poner una buena dosis de perseverancia, empatía, adaptación, tenacidad, innovación y compromiso para continuar mejorando los niveles de satisfacción de los trabajadores en las Compañías y que éstos a su vez, en reciprocidad, hagan compañías rentables, prestigiosas y excelentes lugares para trabajar (Ruyle, 2013).

Existe en la actualidad una historia de más de 50 años de investigación sobre el tema del clima laboral. Numerosos estudios han examinado cómo el clima impacta en una amplia categoría de resultados incluyendo el bienestar psicológico, manejo equitativo (interno y externo) de los salarios y compensaciones, ausentismo, absentismo, rotación de personal, los temas psicosociales de acoso y violencia, comportamientos seguros, resultados concretos, excelente desempeño financiero, administrativo y técnico de una empresa (Litwin y Stinger, 1997).

"Una reciente revisión en varios estudios de clima laboral, de los últimos años, se ha encontrado que el clima laboral ejerce fuertes presiones sobre el desempeño de las personas en relación a los resultados, a lo largo de una amplia gama de entornos de trabajo" (Chiavenato, 1999).

\section{Metodologia.}

Explicación de un caso práctico realizado en una Compañía de Servicios Petroleros en el Ecuador. Luego de haber presentado a la Dirección de la Empresa (objeto de este estudio) los resultados (cuantitativos y cualitativos) del análisis de los factores logrados en la "Encuesta de Clima Laboral" de dicha compañía en el año 2018, se determinó con la Dirección y el Equipo Gerencial, realizar COMO EJEMPLO este nuevo MODELO de mejoramiento sostenido del Clima Laboral, mediante un proceso de intervención terapéutica familiar sistémica a un departamento técnico de 25 trabajadores y sus familias de origen, que 
laboran en el Oriente Ecuatoriano bajo un esquema de horario de trabajo especial, 14 días de trabajo oriente y 7 días de descanso Quito, 14/7(Bradford y Silverstein, 1998).

Con estos antecedentes, se procedió a realizar este modelo de Intervención Terapéutica, en donde, el primer paso fue la creación del programa PISEFA,

\section{Programa Pisefa:}

Sus siglas significan (Programa de Intervención Sistémica Familiar para Empleados y sus Familias). Se trata de una propuesta de INTERVENCIÓN TERAPEUTICA FAMILIAR, relacionada con la necesidad de los trabajadores de mejorar su situación personal-familiarempresa, mediante el diagnóstico, análisis, monitoreo y "mejora continua" de sus situación personal-familiar, que luego en el mediano plazo, este proceso terapéutico incidirá en el mejoramiento continuo de su situación familiar y por ende del clima laboral de la compañía, teniendo como herramienta fundamental, la intervención familiar con enfoque sistémico.

\section{Herramientas del Programa:}

El PISEFA busca integrar las prácticas de la psicología organizacional y la psicología clínica en la Organización, para lo cual utiliza como primer paso la "medición del clima laboral de la compañía", (obtener la primera fotografía del clima de la Empresa), esta es una herramienta técnica utilizada por la psicología organizacional para conocer el medio ambiente humano y físico en el que se desarrolla el trabajo cotidiano de una empresa, el clima laboral está determinado por una serie de factores como por ejemplo: (medición de liderazgo, trabajo en equipo, sentido de pertenencia, comunicación, esquemas salariales, beneficios, entre otros factores similares), para realizar la medición del clima laboral se pueden utilizar las siguientes herramientas (Palom, 2015):

- Utilización de encuestas (físicas y on-line) para la medición del clima laboral.

- $\quad$ Entrevistas individuales o de grupo (grupo focal).

- Análisis estadístico (cualitativo y cuantitativo).

- Informe por factor analizado y colocar las recomendaciones y "oportunidades de mejora continua".

- Comunicar a todo el personal de la empresa, al inicio de la encuesta, sobre la importancia de obtener información fidedigna de este proceso y al final de la encuesta, presentar la información clave de los resultados del proceso de Clima laboral para buscar el compromiso genuino de todos, en las oportunidades de mejora continua.

- $\quad$ Finalmente, se debe aplicar el proceso de Clima Laboral, que puede ser cada 6 meses o cada año, para evaluación y seguimiento (al inicio y final de la aplicación del programa PISEFA), en donde se observará los resultados concretos de mejora continua y el aporte de dicho programa en el proceso de mejorar el Clima de la Organización. 
La segunda herramienta clave en el PISEFA es la concienciación de los trabajadores (con talleres y cursos de formación y capacitación), sobre la importancia de mejorar los procesos administrativos, técnicos y emocionales, en la vida cotidiana de las organizaciones. Se hace necesario, generar un nuevo pensamiento sobre la psicología y la familia, ya que las personas, en este siglo cada vez menos, tienden aún a creer que la intervención psicológica es "para la gente que está loca", para lograr esta concienciación en las personas se propone como subherramientas una serie de talleres de carácter socio-educativo e intrafamiliar, a la vez que estos talleres sirven para que la gente reconozca sus propios problemas familiares y decida buscar asesoría/intervención técnica terapéutica, los mismos que están orientados a mejorar las relaciones familiares e interpersonales que inciden en el clima laboral de la empresa (in situ). Los temas de los cursos-talleres más relevantes que abarca la intervención terapéutica familiar del PISEFA, son:

- Intervención en crisis.

- Intervención terapéutica personal, parejas y familia nuclear (esposo/a e hijos).

- Escuela para padres.

- Manejo de equipos reflexivos.

- Comunicación y trabajo en equipo.

- Dinámicas de grupo, assessment center.

- Talleres de educación, formación, comunicación familiar y su contexto.

- Orientación Vocacional.

- Manejo de casos especiales que requieran nuestra intervención y experiencia (Adicciones, accidentes laborales, intentos de suicidio, depresión, temas de comunicación y relacionales).

- Otros temas de carácter psicosocial, emocional, salud y seguridad.

La tercera herramienta del programa es la intervención y asesoría, a través terapia familiar sistémica para empleados y sus familias (con un aproximado de entre 6-10 sesiones por cada miembro del sistema familiar nuclear). Presentación de informes sobre los avances del proceso de intervención terapéutica familiar del colaborador y su familia, sin colocar nombres, ya que son procesos confidenciales y que de ninguna manera debería afectar la relación laboral con la empresa.

En vista de que el trabajo de intervención sistémica se hace en un contexto organizacional y por lo tanto la empresa necesita conocer las transformaciones que el programa PISEFA provee, este programa contiene (como mencioné anteriormente), una cuarta herramienta.

La presentación mensual de informes gerenciales, (de carácter MUY confidencial, sin presentar los nombres de los colaboradores que se encuentran en intervención, más bien, se incluyen los casos presentados y detallando las conflictivas psicológicas-emocionales abordadas y el proceso sostenido de mejora continua de dichos casos). 
Como última herramienta del programa PISEFA, se encuentra, una presentación delanálisis estadístico general comparativo de la medición de Clima Laboral de la Compañía en el transcurso de un año, en este caso (2017 y 2018), se observa los avances del programa y una propuesta de mejoramiento continuo, esta herramienta está en capacidad de probar cualitativamente y cuantitativamente la eficacia de este estilo de intervención terapéutico dentro de las Organizaciones y su impacto en el Clima Laboral.

\section{Tiempo de duración del programa}

El programa PISEFA es factible realizarlo durante un año calendario, para evidenciar su eficiencia en el esquema y etapas planteadas y que está descrito anteriormente. Mi recomendación, se debe convertir en un programa de apoyo permanente.

\section{Análisis de datos cuantitativos y cualitativos:}

Análisis e Informe del Primer Proceso de Medición del Clima Laboral en la Compañía del Sector Petrolero.

En la investigación de CLIMA LABORAL realizada por el departamento de Talento Humano con los colaboradores de la Compañía, se evidenció que éstos son los tres aspectos fundamentales que determinan: estabilidad, equidad y armonía en la Organización.

a) Relación y Sentido de pertenencia con la Compañía.

b) Relación y Confianza con los líderes de la Compañía.

c) Manejo de las relaciones interpersonales con pares y sus equipos de trabajo.

\section{CLIMA LABORAL (Los factores que fueron evaluados)}

1. Satisfacción laboral

2. Comunicación

3. Actitud y cambio

4. Solución de Conflictos

5. Toma de Decisiones.

Análisis y metodología de la encuesta que se aplicó para medir el Clima Laboral de la Compañía del Sector Petrolero.

- Encuesta personal respondida por los colaboradores, que permite conocer a Talento Humano su percepción (sentir personal-emocional sobre la empresa). Además, sobre el tipo y calidad de las relaciones en su lugar de trabajo, así como los sentimientos que le produce las diferentes facetas de la Compañía. 
- Es un cuestionario escrito que responden los colaboradores de la compañía, en el que se indaga sobre las prácticas de la administración en la gestión de las personas.

- La encuesta contiene 59 preguntas en una escala del (1 a 4) que presenta las siguientes opciones:

1. Nunca Regular

2. Rara vez Buena

3. Frecuentemente Muy Buena

4. Siempre Excelente

Las respuestas, se presentan en porcentajes con respecto al total de la población, se consideran así:

Respuesta 1: El colaborador no tiene una percepción positiva del aspecto considerado.

Respuesta 2: El colaborador percibe inconsistencia en el aspecto considerado o se encuentra indeciso o apático.

Respuestas 3 y 4: El colaborador tiene una percepción positiva y excelente del aspecto considerado.

\section{Análisis Cuantitativo y Gráfico del Clima Laboral en la Compañía del Sector Petrolero años 2017- 2018}

Informe del "Análisis Comparativo" de los resultados de la Encuesta de Clima Laboral, luego de ser aplicado el programa PISEFA, se observa la efectividad de este nuevo MODELO, en un año (2017-2018) se incrementaron alrededor de cinco puntos de satisfacción del Clima Laboral versus el promedio de los factores analizados en la Compañía.

Campos Evaluados

\section{Tendencia}

Satisfacción laboral

Comunicación

Actitud y Cambio

Solución de Conflictos

Toma de decisiones

General
Prom.2017 Prom. 2018 Valoración

$\begin{array}{lll}66 \% & 76 \% & \text { Lig. Superior, Sube } \\ 42 \% & 42 \% & \text { Media, Normal } \\ 54 \% & 55 \% & \text { Media, Sube } \\ 48 \% & 56 \% & \text { Media, Sube } \\ 34 \% & 42 \% & \text { Lig. Superior, Sube } \\ 49 \% & 54 \% & \text { Media, Sube }\end{array}$


Análisis cuantitativo general de la encuesta de clima año 2017 (Se tomó al 80\% de los colaboradores de la compañía, valoración media y con tendencia a mejoramiento continuo).

ANALISIS CUANTITATIVO GENERAL DE LA ENCUESTA DE CLIMA Año 2018 (Subió aproximadamente 5 puntos el clima de la compañía, en relación al año pasado 2017. Valoración media y con tendencia a mejoramiento continuo).

Aceptación $2018 \quad$ Valoración Tendencia

$54 \% \quad$ Media, Sube

\section{Informe comparativo (años 2017-2018) del mejoramiento del clima laboral}

Luego de obtener los resultados cuantitativos de la encuesta de Clima Laboral, se establece cuatro prioridades para trabajar entre el departamento de Talento Humano y el Equipo Gerencial de la compañía. Adjunto dichas prioridades.

Como se ha podido observar en esta comparación entre el modelo clásico de análisis de clima laboral por encuestas y el nuevo modelo que se propone en este artículo, utilizando una herramienta totalmente innovadora y potente para mejorar el Clima Laboral en una Empresa (La terapia familiar dirigida a colaboradores y sus familias) (Orbegoso, 2010).

\section{Contextualización:}

Dónde se realizó esta investigación?

Esta investigación se realizó en una Compañía de Servicios Petroleros, en los años 2017 y 2018. Su especialidad es: sistema de levantamiento artificial por bombeo hidráulico, ingeniería de petróleos, diseño y construcción de equipos y herramientas de fondo especiales para la industria petrolera. Tiene oficinas en Quito y una Planta de Producción para las operaciones con sus clientes, ésta se encuentra en el Coca, Ciudad, Francisco de Orellana en la Amazonía Ecuatoriana.

Por qué resultan importantes en esta investigación los horarios de trabajo especiales establecidos en el Oriente.

\section{Horarios especiales de trabajo:}

Jornada de Trabajo: 14 días seguidos

Días De Descanso 7 días seguidos, contados a partir del día $15^{\circ}$

En las plantas de producción se ha establecido el siguiente horario rotativo de trabajo: 
Primer turno

$7 \mathrm{~h} 00$ a $17 \mathrm{~h} 00$.

Segundo turno

$16 \mathrm{~h} 00$ a $01 \mathrm{~h} 00$.

Tercer turno

$22 \mathrm{~h} 00$ a $07 \mathrm{~h} 00$

Desde la perspectiva sistémica, se demuestra que la gestión humana es también interacción e interrelación con otras personas y que no se puede comprender en este caso al trabajador si se lo aparta de su contexto familiar, social y laboral. Por esta razón, es importante conocer el nivel de impacto que causa esta política (horarios de trabajo) de la compañía, referente a los horarios de trabajo "normales" en la ciudad de Quito.

No es lo mismo tener un horario de trabajo "normal" de 8 horas diarias (08:00h a 17:00h) y de lunes a viernes como sucede con el personal que trabaja en las oficinas de Quito, que tener un horario de trabajo en el Oriente, lejos de sus familias, de 12 horas diarias y de 14 días de labor permanente en actividades de campo, versus 7 días de descanso en Quito.

Este último horario, podría ser un factor CLAVE de esta investigación, al conocer cómo afecta al trabajador y al sistema familiar en esta Compañía de Servicios Petroleros. Por lo tanto, será posible e interesante demostrar de manera cualitativa y cuantitativa, sobre la hipótesis planteada.

"Si las Organizaciones cuentan con personal que provengan de sistemas familiares más funcionales, estables y organizados, entonces el clima laboral de dicha Organización mejorará de manera importante".

\section{Resultados.}

La compañía de servicios petroleros objeto de este estudio, luego de la aplicación del programa PISEFA, se torna en un buen ambiente laboral evidenciado en el nivel satisfactorio en que se encuentran varios indicadores obtenidos del informe final de la medición del Clima Laboral.

Se destaca la percepción referida a la "estabilidad en el trabajo" con una valoración importante.

Adicionalmente, se evidencia un consenso positivo alrededor de la variable alusiva al tema "orgullo de pertenecer a la empresa". Por otro lado, se debe reforzar más sobre el tema remunerativo, encontrándose muy bien en varias posiciones acorde al sector de servicios petroleros. Convenientemente gestionada y equilibrando otras posiciones más bajas en la remuneración, podría llegar a ser una fortaleza de la compañía. En este tema hay que trabajar más proveyendo información técnica y objetiva de mercado.

En el norte de la organización se ve perfectamente factible continuar avanzando hacia resultados más llamativos donde sea posible consolidar mayores fortalezas. Para esto será 
necesario trabajar sobre las prioridades identificadas, desarrollando estrategias que las favorezcan con fortaleza y compromiso a todos los actores de la Compañía.

\section{Conclusiones.}

Frente a estas prioridades como acciones de mejora continua están:

- Implementar un sistema de ejecución ligado a programas sostenidos de capacitación en: "comunicación”, "empoderamiento", “delegación”, "liderazgo", "trabajo en equipo", "manejo efectivo del tiempo", "observación y escucha", etc. Este tipo de intervención puntual seguramente no resolverían los problemas de fondo, con lo cual en poco tiempo se haría necesaria otra intervención terapéutica familiar de "mayor impacto" tomando en cuenta los aspectos psicosociales y familiares del personal. Sin embargo, lo que hasta aquí se ha realizado es el sistema clásico de funcionamiento de los departamentos de Talento Humano en las empresas con respecto a mejorar el clima laboral de las empresas con una gran dosis de pensamiento SISTEMICO.

- Manejar los procesos con una gran dosis de empatía, (p.ej. liderazgo de apoyo)

- Claridad absoluta en la información a todos los niveles de la compañía, (p.ej. compartir y entender claramente los objetivos a seguir)

- Fortalecer al interior de la compañía los procesos de compromiso, (p. ej. integrar a las personas en el proceso de toma de decisiones, relaciones interpersonales, alineación con los objetivos)

- Desarrollar programas formales de capacitación, educación, entrenamiento y aprendizaje continuo, (p. ej. apreciación, devolución, capacitación y procesos de desarrollo).

- Ideal, previo proceso interno con un profesional de bienestar como la trabajadora social, ésta debería referir a un profesional psicólogo, para realizar procesos terapéuticos, (p. ej. Apoyo terapéutico familiar puntual por problemas derivados del trabajo).

- Finalmente, bajo el criterio de salud, higiene y seguridad industrial, el trabajo planificado y organizado en estos procesos de seguridad, higiene y salud organizacional, se ha descubierto que los equipos de trabajo en donde estos elementos funcionan óptimamente exhiben el menor riesgo de comportamientos perjudiciales por parte del trabajador.

\section{Referencias bibliográficas.}

Andolfi, M. (1977). La terapia con lafamiglia. Roma: Astrolabio Ubaldini editore. Versión cast. (1994). Terapia familiar. Buenos Aires.

Beck, J. (2000). Terapia cognitiva. Barcelona: Gedisa.

Bradford, P. \& Silverstein, O. 1988. “La Voz Terapéutica de Olga Silverstein”. Ed. Piados. Buenos Aires.

Centro Walmart 2014. Conciliación Familia y Empresa: un nuevo pacto es posible. (Centro Walmart Conciliación Familia y Empresa del IAE Business School, en alianza con Walmart Argentina, presentaron en Rosario la "Guía de Buenas Prácticas: Hacia un nuevo pacto entre el trabajo y la familia"). 
Chiavenato, I (1999) Administración de Recursos Humanos. (2da Edición). Bogotá, Colombia: McGraw-Hill.

Fineman, S. (2000). Emotion in Organizations. New York: Sage.

Guidano, V. (1996) El sí mismo en proceso. Barcelona: Paidós.

Juape Miguel, 2014. Buenas prácticas laborales incrementan la productividad de la empresa en un $30 \%$

Kim Ruyle, 2013. Vice Presidente de Desarrollo de Productos en Leadership \&TalentConsultig de Korn/Ferry International. Las mejores prácticas en gestión del talento.

Litwin, G y Stinger, H. 1997.“Dimensiones del Clima Organizacional”. Sociedad Latinoamericana para la calidad. Internet.

Maturana, H. y Varela, F. (1984). El árbol del conocimiento. Chile: OEA.

Minuchin, S. “Familias y Terapia Familiar”. Ed. Gedisa. Argentina- Buenos Aires. 1986.

Morris, Charles y Maisto, Albert, (2009), Psicología, Pearson, México.

Orbegoso Galarza Arturo, 2010.Revista Psicológica. Clima

Laboral:http://www.climalaboral.com.es/

Palom, Santiago, 2015. Art. “Cómo medir y Mejorar el Clima Laboral” España (Director General de Grupo ODE. Consultor Senior en Management y Recursos Humanos.

Reeve, Johnmarshall, (2009), Motivación y Emoción, Mcgraw Hill, México.

Santillán, W. (2017). Nuevas prácticas para mejorar el clima laboral. Quito: Don Bosco.

Toro, F. (Ed.) (2010). Clima Organizacional, una Aproximación a su Dinámica en la Empresa Latinoamericana. Segunda Edición Medellín: Editorial Cincel.

\section{LCiencia}




\section{PARA CITAR EL ARTÍCULO INDEXADO.}

Santillán Marroquín., W., Paredes García, H. E., Garcés Uquillas, G., \& Santillán Vieira, J. (2019). La psicología y su influencia en el clima laboral de las empresas. Ciencia Digital, 3(3.2), 290-301. https://doi.org/10.33262/cienciadigital.v3i3.2.757

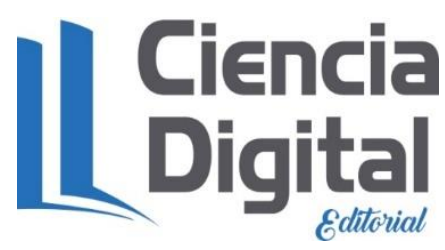

El artículo que se publica es de exclusiva responsabilidad de los autores y no necesariamente reflejan el pensamiento de la Revista Ciencia Digital.

El artículo queda en propiedad de la revista y, por tanto, su publicación parcial y/o total en otro medio tiene que ser autorizado por el director de la Revista Ciencia Digital.
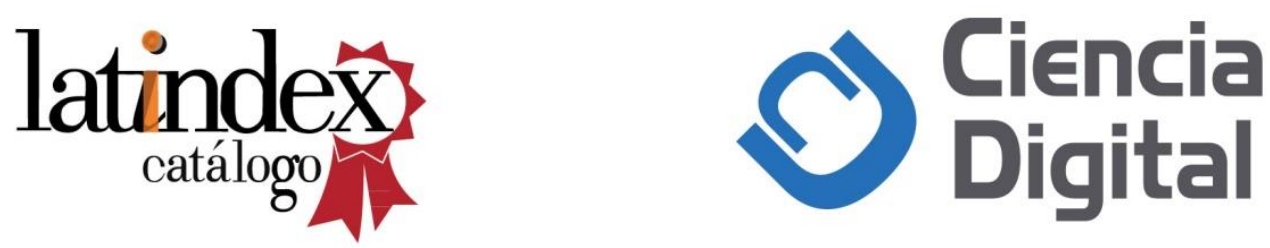